\title{
Discussion on Aesthetic Marketing in the Age of Sensibility
}

\author{
Meijin Hsiao $^{1, *}$ Shuhua Hsueh ${ }^{1}$
}

\author{
${ }^{1}$ Fuzhou University of International Studies and Trade, Fuzhou, Fujian, China \\ *Corresponding author. Email: 1601120303@qq.com
}

\begin{abstract}
Under the pressure of global industrial competition in the 21st century, perceptual design has become a prominent subject in the field of design. With the progress of science and technology and the improvement of people's life quality, modern consumers have gradually become more and more sensitive to the demands of products. It is an important subject for modern designers to design products more in line with consumers' demands through Kansei Engineering. Through the discussion of relevant literature data, this paper sorts out the theories and methods of Kansei Engineering and the practice and application of perceptual design. Firstly, this paper discusses the fashion significance of the Age of Sensibility, describes the theories, types and methods of Kansei Engineering, and the relationship between Kansei Engineering and fashion. Secondly, it explores the perceptual aesthetic style, describing that style is composed of aesthetic elements such as shape, color and texture. Third, the paper focuses on the marketing and strategy in the Age of Sensibility, aiming to point out that consumers' motivation to buy goods is not just the use function of goods. Aesthetic marketing strategy is to establish a series of aesthetic marketing strategies based on the five senses (vision, hearing, smell, taste and touch) of the perceptual experience generated in the enterprise brand. Fourth, the paper expounds that designers should grasp the sense perception and psychological needs of customers, systematically implant the user's needs into the prototype design, and make the perceptual design the key to adding value of creative economy, aesthetic economy and knowledge economy.
\end{abstract}

Keywords: aesthetic marketing, aesthetics, style

\section{INTRODUCTION}

\section{A. Kansei Engineering theory}

Kansei is originated from Japanese, which means perception, feeling and impression. It is a psychological feeling generated by consumers towards products. It is information processing through individual thinking, which includes the judgment of psychological function, cognition, and then produces a variety of comprehensive perceptual consciousness. Therefore, the development of design products must be influenced and even changed by various internal and external environmental factors. ${ }^{1}$ These factors are mainly targeted at people, that is, the needs of the five senses (vision, hearing, touch, taste and smell) generated by the change of people's views on various things. Kansei Engineering was first proposed and marketed overseas by Kenichi Yamamoto. In 1970, Nagamachi believed that the future would usher in the Age of Emotion, and

Wang Mingtang. Kansei Engineering to Kansei Design- the Basic and Application for Researching of Kansei Engineering. Taipei, p26, 2016. when human beings are rich in material resources, the pursuit of spiritual satisfaction would follow. Therefore, he proposed "Emotion Technology". Considering foreigners' understanding of emotion, in 1988, "Emotion Technology" was renamed "Kansei Engineering".

The spiritual connotation of Kansei Engineering is consumer-oriented, and its role is to assist designers in the development of products. Kansei Engineering can transform customers' feelings towards products into technologies of design elements. Further, through the technical methods of Kansei Engineering, people can find out the image and feeling of products and then transform this image and feeling into the reference elements for designers to design products. In addition, perceptual analysis of people can also be conducted through systematic program patterns or scientific methods such as quantification, which can then be applied to the design of goods that make people happy and satisfied. ${ }^{2}$ In general, Kansei Engineering is the

\footnotetext{
2 Nagamachi, M. (1995a). Kansei Engineering: A New Ergonomic Consumer-Oriented Technology for Product
} 
application of technology of Kansei Kngineering to explore the relationship between human sensibility and the properties of matter as the basis for design. The purpose of Kansei Engineering is to design new products according to consumers' feelings and needs, and to make the design elements of the designed products correspond to people's feeling. By means of Kansei Engineering, people's emotional feelings are quantified, so that designers will know which design elements can better meet people's perceptual awareness.

However, to understand the needs and thoughts of consumers, one must first understand how people perceive things. The reason for the purchase motivation is that the product itself meets the needs of consumers from the perspective of rationality and sensibility. The rational needs can be satisfied by the product's function, environment and use, while the perceptual needs can be satisfied by design, impression and other factors. If these two aspects are combined, consumers can find the value of the product and then generate emotional behavior of emotional purchase. And the so-called sensibility is the value consciousness, personality, preference and the meaning of things that touch people's hearts.

\section{B. Research on the types of Kansei Engineering}

Nagamachi proposed six methods of researching Kansei Engineering as follows. ${ }^{3}$

- Category Classification of the perceptual concepts: that is to determine the concept of the initial product development, conduct decomposition of the physical quantity obtained by the conceptual analysis.

- Kansei Engineering System: On the basis of expert system, the calculation of database and reasoning engine is implemented in several computers to produce the method suitable for consumer's emotional design.

- Hybrid Kansei Engineering System: It is the "forward Kansei Engineering" that uses computer design to systematically carry out the diagnosis of Kansei Engineering, and produce design results with the creativity of computers.

- Kansei Engineering System Modeling: The mechanism of Kansei Engineering is constructed by mathematical methods.

- Virtual Kansei Engineering System: Experience is stimulated by means of Kansei Engineering

Development. International Journal Industrial Ergonomics, 15(1), P311.

Wang Mingtang (2016). Kansei Engineering to Kansei Design- the Basic and Application for Researching of Kansei Engineering. Taipei, P158-159, 167, 174-175. design. It means to create a space for computer virtual reality technology and enter it and experience this, that is, to combine Kansei Engineering and virtual reality to develop a computer system for consumers' individual perceptual adaptation and product development.

- Collaborative Kansei Engineering: it is also known as the design supporting system for network perceptual group (Internet) - placing the design produced by the three-dimensional design software in the server of the network, and then using the database and design to support the distance between designers, and make them discuss with each other to design the computer system, is commonly known as the concept of collaborative design.

With the development of economy and the improvement of people's life quality, the demand for fashion is becoming more and more exquisite. Xingye Kemei, who proposes the new "consumer culture phenomenon", argues that consumer action is not limited to "consumption of things", but has been transformed into a cultural act of "sensibility and imagery" about goods, and their lifestyles meet their needs from an emotional point of view. In addition, Yoshiko Yoshida further proposed that the post-modern consumption structure has been oriented towards the deepening of the value of life, and the desire for life has expanded to the intrinsic value, including the entire psychological and symbolic life world. In the $21 \mathrm{st}$ century, people are gradually pursuing a form of lifestyle fashion, no matter in the spiritual level or in the material and visual sense. From the tangible external aspects of daily life, such as food, clothing, housing, transportation, education and music, to the intangible inner part of the soul, everyone is full of the pursuit and yearning for perceptual life. Fashion can be said to be a kind of spiritual feeling and material form of concrete expression. When people have enough to eat and wear, they will certainly pursue higher enjoyment in life. In fact, the development of fashion culture is diversified and extensive. Art, dance, music, film, literature, culture and industry are all important elements indispensable for people in modern life. Therefore, fashion is the attitude of life. It permeates the whole picture of life, and it is embodied in people's daily life.

\section{THE AESTHETIC STYLE OF THE AgE OF SENSIBILITY}

Aesthetic feeling is a sense of beauty judgment, the innate nature of human beings, and modernity is a ubiquitous word. "Aesthetics" comes from the inner or external feeling of beauty. In terms of "products", "industrial conditions" and "living conditions" must be combined with each other. According to Newman, a 
product is a symbol formed by form, size, color, and function, and this symbol may have different importance and influence depending on its relevance to individual needs and social interactions. Thus, a product is the sum total of the meanings it transmits, consciously or unconsciously, when people are looking at it or using it. ${ }^{4}$ (Newman)

With the plebification of style and taste in recent years, everyone has his own idea of beauty. Style, taste, and judgment of "beauty" all show one's own philosophy of life. For example, "high-quality goods" are no longer a class, but can be used by everyone to enjoy life and make themselves pleasing to the eye. In modern culture, commerce, technology and design, as well as daily necessities, color, shape and texture, are all reflected in food, clothing, housing, travel, education, music each level, with more rich and diverse design styles, all strengthening the importance of perceptual design.

Style can create brand awareness and trigger all kinds of mental and emotional connections. Style can distinguish products and services from others and help customers classify related products and services. For the whole society, style can beautify the surroundings and distinguish the areas for leisure and relaxation. Style is a kind of identification carried out by aesthetics. A style is a distinctive trait or formation, a way of expression. Style is a constant form of artistic expression by an individual or group, sometimes is the constant elements and modes of expression. It covers fashion design, interior design, furniture, architecture and food. Therefore, the design style of life products has become a symbol of a culture or lifestyle.

"Style" is not only the operation and means for attracting consumers in marketing strategy, but also the responsibility and mission of enterprises in product development. In the societies of developing countries, people already have a considerable level of knowledge and living standards, it is impossible for enterprises or products without a unique style to leave any impression in the minds of consumers.

The word style was used by Greek philosophers from Aristotle to Cicero to praise the orator's habit of using "words" and "sentences". Count Buffon of France thought "Style is the person himself (Le style est 1'homme même)." "Style is the Physiognomy of the mind," said Arthur Schopenhauer of Germany. It is the external manifestation of the inner mind. Therefore, in terms of products, the style of products is a kind of value judgment between enterprises and customers. Enterprise style is the organizational spirit of the enterprise, which is more reflected in architecture, product design, advertising and corporate identification

4 Newan, J. W (1957). New Insights, NewProgress, for Marketing, Harvard Business Review, Nov. - Dec., P100. system (CIS), etc., so enterprise style is also the perceptual level of aesthetics.

Style is composed of many major aesthetic elements. Color and shape, line and form are the key elements of "visual" style. Consumption is an experience of multiple senses. Basic elements such as a retail store or a scene have to be managed. When customers enter a department store or boutique, the setting and sound, material and texture of the background will have an impact on customers. The main elements of style include sight, hearing, taste and smell. Especially in the category of marketing aesthetics, vision is the most important element in the style, which also includes color, shape, material and texture. The Greek philosopher Aristotle once said that "all cognition is caused by seeing", which is the best portrayal of companies and brand recognition. According to psychological research, people will have a considerable memory for the image; with the text, the image will have a higher degree of uniqueness, and therefore can be maintained in people's memory for a long time. Taking the example of memory experiments of separate texts, separate images and a combination of both, the result is that patterns with pictures and texts are the most impressive, as words produce a high degree of recognition, images are complex stimuli with a high degree of specificity, and the combination of the two is the most memorable. From the point of view of marketing aesthetics, the main visual element is the form of color, and the font is also one of the important items, which often appears in the brand name, packaging, advertising, promotion activities, brochure, product catalog, business card, stationery, writing paper and so on.

The appearance of the package has a great influence on the product. "Appearance" can also become a trademark or an image, and appearance is also a kind of recognition. Most customers usually have an immediate feeling and imagination for the appearance and packaging of each product. Such recognition of the appearance of the package triggers customers' memory and contributes to the marketing effect of the product. Appearance is an aesthetic element. The appearance of a single case can be said to be the logo of the enterprise and the trademark of the enterprise, which related to the success or failure of product marketing. Therefore, efforts should be made in the design to show its charm and cope with the marketing advertising campaign in the process of prominent image. The shape is different from the corporate name, and it crosses cultural boundaries more easily, impressively and fondly. In an aesthetic strategy, the critical aspect of the product's shape in the design should be angle, symmetry, proportion and matching of dimensions.

In the enterprise and brand identification, color is everywhere. The enterprise logo is usually displayed in 
color, and various products will also be in different colors. Packaging appeals to consumers because of the different colors they present. "Color" is the confirmation of the enterprise and the brand. Any company can use a particular color or combination of colors as part of its visual identity, making it the main focus of its identification, and making color its trademark. For example, the green logo of Starbucks and the red identification of Coca-Cola are all practical cases in this respect. As for the company, it not only copies the color on products all over the world, but also protects the color very much. The business community also uses color to distinguish various products into different categories of items, as different product category identification. For example, the Starbucks style has set off a storm in various countries and regions, becoming a synonym for relaxing time, coffee tasting and leisure enjoyment. When people talk about Starbucks, they associate it with places for gathering, chatting and relaxing. In order to meet the needs of local customs and markets, Starbucks in each location will vary with local customs. The brand style of Starbucks adopts the traditional green image of a longhaired woman and signs with a natural style in burlywood, brown and white, etc. ("Fig. 1"). Starbucks' style attracts customers mainly because of its success in shaping the perceptual aesthetic style. The warm, relaxed field brought by the strong coffee aroma (taste and smell) as well as vision, constitutes the charm of Starbucks style ${ }^{5}$ ("Fig. 2" and "Fig. 3").

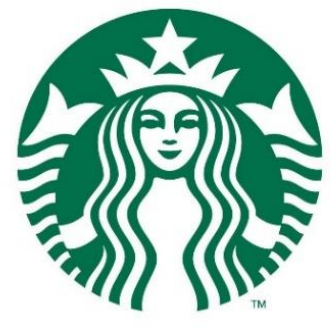

\section{STARBUCKS}

Fig. 1. Green image of a woman with long hair.

${ }^{\text {a. }}$ Source: Shot by the author.

Bernd H. Schmitt, Alexander Simonson (1999). Marketing Aesthetics. Taipei. P33, 35, 95-100.

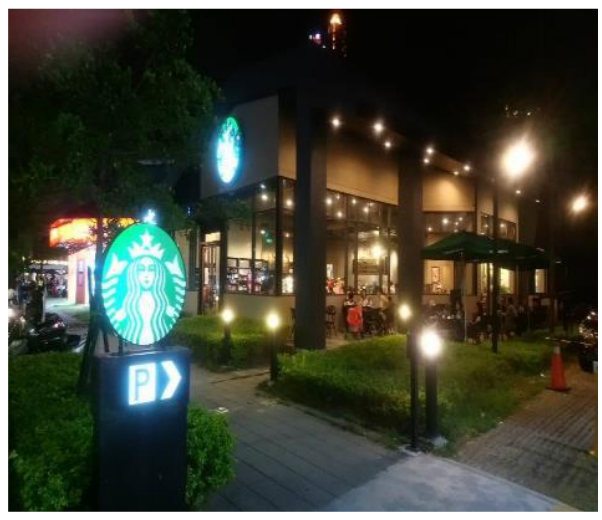

Fig. 2. Starbucks.

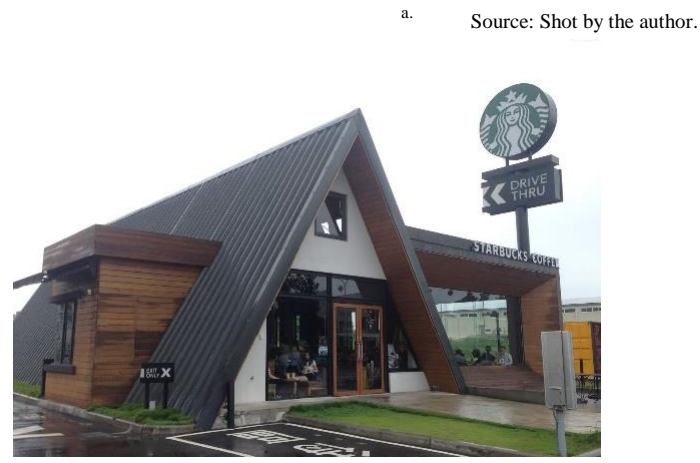

Fig. 3. Starbucks.

Source: Baidu

\section{AESTHETIC MARKETING AND STRATEGIES}

Appearance and color enrich the human sensory world. When people open their eyes, they are influenced by everything they see, hear, and feel. With the progress of science and technology and the improvement of life quality, modern consumers gradually become more and more sensitive to the demand for products. When consumers buy a product, their motivation is not only the "use function" of the product, and practicality is no longer the only choice for them. In terms of modern consumption phenomenon, the shopping desire of the middle class is attached to the "symbol" of goods. Therefore, in addition to the value that the product brings to the consumer, the product becomes a projection of the consumer's heart. The reason why the product can produce consumption function is that it meets the level of the intrinsic value of consumers. This means that the society has gone beyond the original level of consumption to maintain life, and began to add cultural and emotional factors, and even appeared irrational tendency, which is of unique significance value for affluent and mature consumers. Taking the "LV" bag from France, which is popular all over the world for example, the high price paid is by no means for the capacity and use, but the coffee grid and LV brand logo with different shades. 
Another example is Absolut Vodka. In each advertisement, whether it is a print AD design or an art deco, its unchanged bottle and letter style provides a unique look that is configured in a variety of settings to demonstrate its unique aesthetic. When the popularity of commodities transforms from the advantages and disadvantages of "use function" to the competition of "perceptual function", the focus of commodity development shifts from the reasonable focus of manufacturing process and cost to the economic field of product packaging and aesthetic marketing of industry design. ${ }^{6}$

Aesthetic marketing is associated with experiencing a brand or a business style. Taking the aforementioned Starbucks and Absolut Vodka as an example, they provide consumers with an unforgettable emotional experience, which is derived from the color, shape, texture, taste and touch of the external visual sense. Only in this way can modern consumers enjoy such an unforgettable experience. Therefore, aesthetic marketing can provide tangible and intangible value for the enterprise - it can create loyalty for the enterprise, and undoubtedly bring high prices and profits for the enterprise ("Fig. 4", "Fig. 5" and "Fig. 6") .

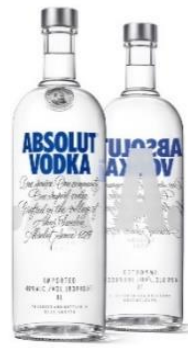

Fig. 4. The bottle look of Absolut Vodka.

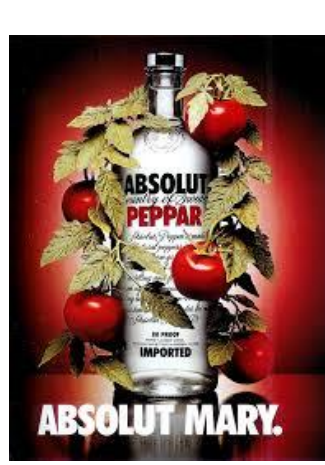

Fig. 5. The bottle look of Absolut Vodka.

6 Zhan Weixiong (2005). The Economy of Aesthetics: 60 Micro-perspectives on Transition of Taiwan. Blue Whale Publishing, Taipei. P25, 69, 71.

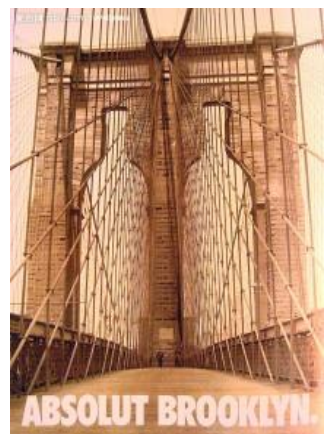

Fig. 6. The installation art of Absolut Vodka.

Source: Baidu

\section{Conclusion}

Under the pressure of global industrial competition in the 21 st century, perceptual design has gradually entered the industry and become a prominent subject in the field of design. The progress of science and technology and the improvement of life quality have made modern consumers' demand for products more and more sensitive. In a constantly renewing society, each age is the age of "style aesthetic competition", where only the fittest survive. The consumption behavior of modern economy is not limited to the consumption of things, but pays more attention to the feeling of life, so as to conform to the feelings in the hearts of consumers. Therefore, when a new product is developed, the Kansei Engineering design method can be used to grasp the customer's perception of the product to be launched and make diversified perceptual data analysis so as to obtain the final design of the product. Therefore, in the face of the rational and perceptual consumer market, to use Kansei Engineering design method to design in line with the needs of consumers, and to use aesthetic marketing strategies to create more and higher profits for the enterprise is the major task and challenge faced by modern designers.

\section{References}

[1] Wang Mingtang (2016). Kansei Engineering to Kansei Designthe Basic and Application for Researching of Kanse Engineering. Taipei. P26, 158-159, 167, 174-175.

[2] Nagamachi, M. (1995a). Kansei Engineering: A New Ergonomic Consumer-Oriented Technology for Product Development. International Journal Industrial Ergonomics, 15(1), P3-11.

[3] Newan, J. W (1957). <New Insights, NewProgress, for Marketing, Harvard Business Review>, Nov. - Dec., P100.

[4] Bernd H. Schmitt, Alexander Simonson (1999). Marketing Aesthetics. Taipei. P33, 35, 95-100.

[5] Zhan Weixiong (2005). The Economy of Aesthetics: 60 Microperspectives on Transition of Taiwan. Blue Whale Publishing, Taipei. P25, 69, 71 .

[6] Huang Guangnan (2006), Form Exquisite Craftsmanship tp Cultural Creativity, National Taiwan University of Arts. 
[7] Chen Kuo-Hsiang(2009), Kansei Engineering , Quality Monthly CSQ, 45(4), P21-22.

[8] Gerald Zaltman. How Customers Think: Essential Insights into the Mind of the Market [M]. Trans. Yu Yifang, Good Morning Financial Culture, Taipei, 2004.

[9] He Mingquan (2003). The Power of Dynamic Design, Review and Prospect of 2004 Design Research, National Yunlin University of Science and Technology, P31.

[10] Xiao Rufang (2006). A Study on the Value of Consumer Preferences for life Products - Taking Muji as an Example. Master thesis, College of Fine Art, National Taiwan University of Arts.

[11] Lin, Minghuang (2003). Industrial Design Trend - Design Trend and Product mModeling Series. Chuan Hwa Book Co., LTD., Taipei, 2003.

[12] Roland Barthes, Dong Xuewen, Wang Kuiyi. Semiotic Aesthetics. Shining Culture Publishing Co., Taipei, 1992. 